\title{
Thermally Stable Polyarylenes with Low Dielectric Constant: Direction towards the Lowest Limit of Dielectrics
}

\author{
Kohei Goto*, Toshiyuki Akiike, Keiji Konno, Tadahiro Shiba, Matthias Patz, \\ Masayuki Takahashi, Yasutake Inoue, and Minoru Matsubara
}

\author{
Tsukuba Research Laboratories, JSR Corporation \\ 25 Miyukigaoka, Tsukuba, Ibaraki 305-0841, Japan
}

\begin{abstract}
We successfully prepared a series of soluble, thermally stable polyarylenes with low dielectric constant $(k)$ by coupling polymerization of aromatic dihalides and/or bismesylates of the corresponding bisphenols using $\mathrm{Zn}$ and Ni-phosphine catalysts. Among the non-fluorinated polyarylenes, a soluble copolyarylene derived from bismesylates of 9,9-bis(4-hydroxyphenyl)fluorene and 4,4'-dihydroxyphenyl-diphenylmethane showed with $k=2.7$ (@1MHz), Tg: $>300^{\circ} \mathrm{C}$, and $\mathrm{Td}\left(5 \%, \mathrm{~N}_{2}\right): 549^{\circ} \mathrm{C}$ the lowest $k$ value. The polyarylene with the lowest $k$, a fluorinated polymer prepared from the bismesylate of 2,2-bis(4-hydroxyphenyl)hexafluoropropane, showed $k=2.2$ (@1MHz), Tg:249 ${ }^{\circ} \mathrm{C}$ and Td $\left(5 \%, \mathrm{~N}_{2}\right): 508^{\circ} \mathrm{C}$. Among organic polymers, soluble polyarylenes proved to be very promising as low $k$ materials with high thermal stability. In addition, we show based on these new results and previous results with fluorenyliden-substituted polyimides how to estimate the dielectric constant in polyimides and polyarylenes as a function of polar linkage (imide) concentration and fluorine content.
\end{abstract}

Keywords: polyarylene, low dielectric constant, thermal stability, estimation of dielectric constant

\section{Introduction}

A challenge for thermally stable polymers in the field of microelectronics is lowering their dielectric constant $(k)$, since at high wiring density the signal propagation speed depends on the $k$ values of the interlayer dielectrics between the conducting lines in the devices.

Considerable efforts have been focused in the last decade on high performance polymers, such as polyimides, polyquinoxalines, polyquinolines, etc., to design and synthesize new polymers with lower $k$ for this purpose.

We already reported a series of low $k$ polyimides with bulky fluorenylidene-moieties in the backbone [1]-[4]. The lowest dielectric constant $k$ has been $k=2.77$ (@1MHz) for non-fluorinated and $k=2.35$ (@1MHz) for fluorinated polyimides. It is suggested that these $\mathrm{k}$ values might be the lowest values that can be obtained for non-porous polyimides. Based on these results we expected that polyarylenes without a polar imide linkage might show even lower $k$ values than the polyimides with such moieties.

In this article, we report the synthesis, dielectric constants and thermal properties in a series of soluble polyarylenes prepared by coupling polymerization. These polymers are expected to be promising materials as interlayer dielectrics since they combine low dielectric constants with high thermal stability.

\section{Experimental}

1) Monomer and its Synthesis

Monomers for the polyarylenes were aromatic dichlorides and/or bismesylates of bisphenols. The dichlorides, trifluoromethyl-2,5-dichlorobenzene 
(TFDCB), 1,3-dichlorobenzene and the bisphenols, 9,9-bis(4-hydroxyphenyl)fluorene, 2,2-bis (4-hydroxyphenyl)hexafluoropropane (Bisphenol AF), 2,2-bis(4-hydroxyphenyl)propane (Bisphenol A), bis(3-fluoro-4-hydroxyphenyl)diphenyl methane, bis(4-hydroxyphenyl)phenyltrifluoromethylmethane, bis(4-hydroxyphenyl)sulfone (Bisphenol S), 4,4'-dihidroxybenzophenone (Bisphenol K) and 1,7-dihydroxynaphthalene are commercially available as reagent grade and were used as received. 4,4'-dihydroxyphenyldiphenylmethane was synthesized from diphenyldichloromethane and phenol by nucleophilic substitution. Mesylates were prepared from the corresponding bisphenols by reaction with methanesulfonyl chloride $(\mathrm{MsCl})$ in the presence of pyridine as acid acceptor. The new compound, 9-bis(4-methylsulfonyloxyphenyl)fluorene has a melting point of $220^{\circ} \mathrm{C}$. The structure was confirmed by NMR spectroscopy.

2) Polymer Synthesis

Polyarylenes were prepared by $\mathrm{NiCl}_{2} / \mathrm{PPh}_{3} / \mathrm{NaI}$ catalyzed reductive coupling of the corresponding aromatic bismesylates and/or dichlorides using $\mathrm{Zn}$ in anhydrous dimethylacetamide (DMAc) at $70{ }^{\circ} \mathrm{C}$ for $20 \mathrm{hrs}$ under argon atmosphere [5], [6].

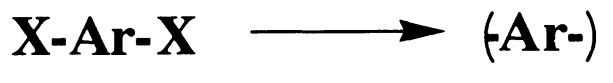

$$
\mathrm{X}: \mathrm{Cl},-\mathrm{OSO}_{2} \mathrm{CH}_{3}
$$

The polyarylenes were coagulated in a large excess of a mixture of methanol and $36 \%$ hydrochloric acid. Purification was performed by repeated precipitation from THF solutions in methanol. Recovered polymers were dried overnight in vacuo at $80^{\circ} \mathrm{C}$.

The chemical structure of polyarylenes was confirmed by IR and NMR spectra.

3) Characterization

Weight-averaged molecular weights $(\mathrm{Mw}) \mathrm{s}$ of the polyarylenes were measured by GPC using THF as solvent. The GPC was calibrated using a polystyrenes standard.

Thermal decomposition temperature at 5\% weight loss (Td) was measured with a TGA 320 (Seiko Denshi Kogyo Co.) at a heating rate of $10^{\circ} \mathrm{C}$ /min under nitrogen atmosphere.

Glass transition temperature ( $\mathrm{Tg}$ ) was measured with a DSC 910 (Du Pont Co.) at a heating rate of $20^{\circ} \mathrm{C} /$ min under nitrogen atmosphere.

For the measurement of $k$ the polymers were spin-coated onto flat conductive substrates (Asdoped Si wafer). The film thickness was measured by ellipsometry (DVA-36LH, Mizojiri Co. Ltd). Aluminum electrodes were deposited in vacuo through a mask on top of the polymer films. All measurements of $k$ were performed in air, at $1 \mathrm{MHz}$ and room temperature using a LCR meter (HP4284, Hewlett Packard Co.). The dielectric constant $k$ was calculated using Eq.1.

$$
k=\frac{\left(C_{x}-C_{r}\right) t}{\varepsilon_{0} \pi d^{2} / 4}
$$

Where $C_{x}$ is the measured capacitance, $C_{r}$ is the calibrated capacitance, $t$ is thickness of the sample, $d$ is diameter of the electrode and $\varepsilon_{0}=8.85 \times 10^{14} \mathrm{C} / \mathrm{m}$. $\mathrm{C}_{\mathrm{r}}$ was calculated according to ASTM D150.

\section{Results and Discussion}

Table 1 shows the composition, Mw, thermal properties ( $\mathrm{Tg}$ and $\mathrm{Td}$ ), and $k$ of the prepared polyarylenes. Table 2 shows the solubility of these polymers.

Generally polyarylenes have a tendency to poor solubility owing to the rigid and conjugated polymer structure even if rigidity is reduced (Xa) (entry No.1 in Tables) or heteroatoms are introduced (entry No.2 and 3 in Tables, monomers from Bisphenol S (Xb) and Bisphenol $\mathrm{K}(\mathrm{Xc})$, respectively).

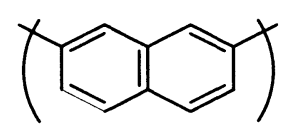

(Xa)<smiles>[Y][Y](c1ccc(CC)cc1)c1ccc(C(C)C)cc1</smiles>

(Xb) $\mathrm{Y}:-\mathrm{SO}_{2}$ (Xc) Y: -CO-

One possibility to increase solubility for polymers is to introduce bulky substituents into the backbone and/or to create irregular structures, e.g. by copolymerization. 
Table 1 Characteristics, Mw, thermal properties and $k$

\begin{tabular}{cccccccc}
\hline Polyarylene & $\begin{array}{c}\text { Entry } \\
\text { No. }\end{array}$ & $\begin{array}{c}\mathrm{F}^{* 1)} \\
(\mathrm{wt} \%)\end{array}$ & $\mathrm{Mw}^{* 2)}$ & $\begin{array}{c}\mathrm{Tg} \\
\left({ }^{\circ} \mathrm{C}\right)\end{array}$ & $\begin{array}{c}\mathrm{Td} \\
\left({ }^{\circ} \mathrm{C}\right)\end{array}$ & $\begin{array}{c}k \\
(@ 1 \mathrm{MHz})\end{array}$ \\
\hline $\mathbf{X a}$ & 1 & 0 & $>5000$ & $>300$ & 485 & - \\
\hline $\mathbf{X b}$ & 2 & 0 & - & $>300$ & 547 & - \\
\hline $\mathbf{X c}$ & 3 & 0 & - & $>300$ & 487 & - \\
\hline $\mathbf{X d}$ & 4 & 0 & $>13000$ & $>300$ & 562 & - \\
\hline
\end{tabular}

Copolymer of Xd with other aromatic monomers

Composition

Comonomer molar ratio

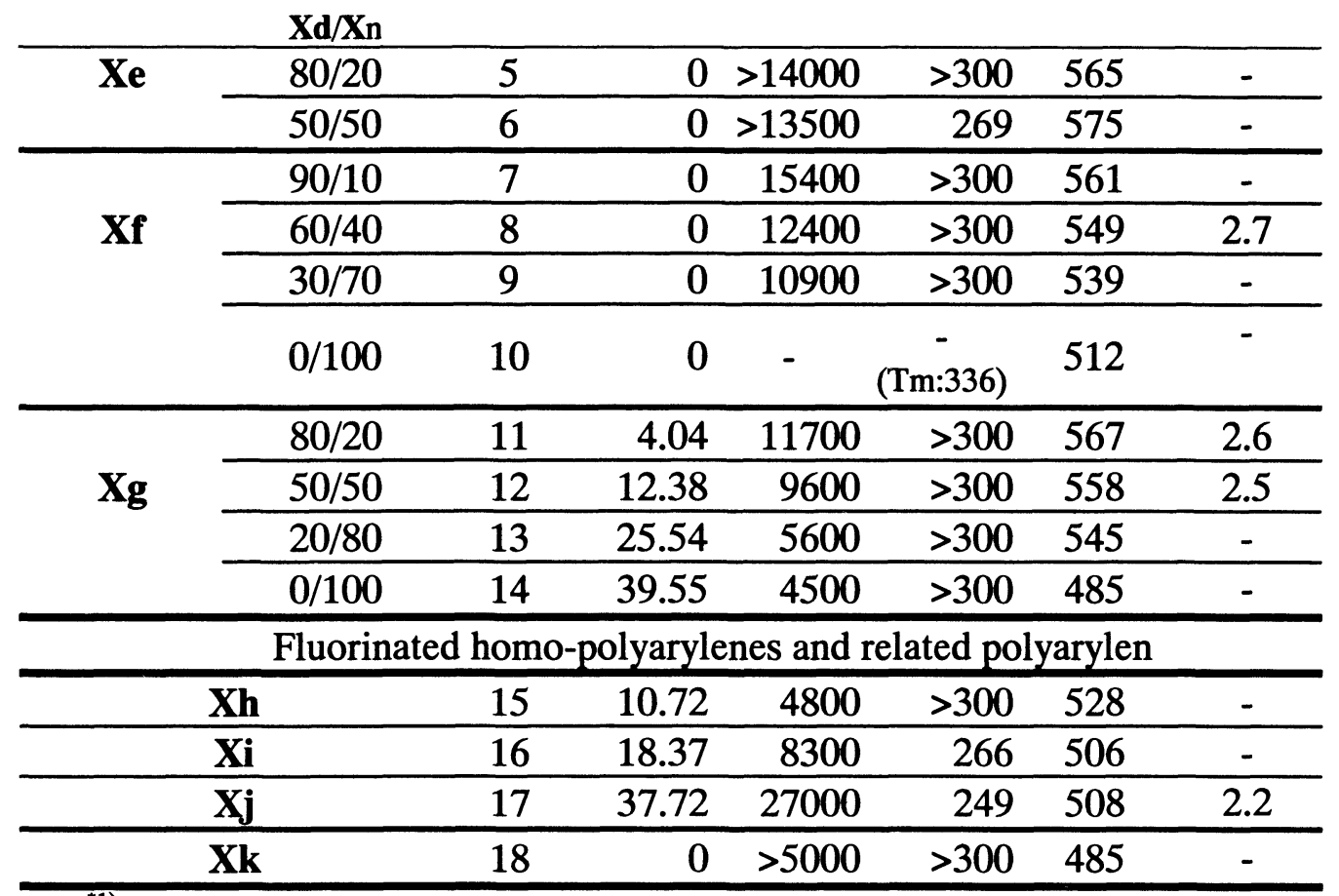

${ }^{\text {"1) }}$ Fluorine content calculated from chemical formula in repeating unit.

${ }^{* 2)}(>)$ means polymer includes insoluble parts of high $\mathrm{Mw}$.

Bulky fluorenyliden-substituents have been shown to be capable of increasing solubility while keeping high thermal stability [7] and low $k$, which was also reported by us for a series of polyimides [1]-[4]. Polyarylenes derived from the fluorenyliden-monomer (Xd) were therefore expected to combine good solubility with excellent thermal properties and low $k$.

High molecular weight poly(Xd) was easily obtained by coupling polymerization of the corresponding bismesylate, but is soluble only in amidic solvents such as DMAc or $\mathrm{N}$-methylpyrrolidone (NMP) ( entry 4 in Tables).

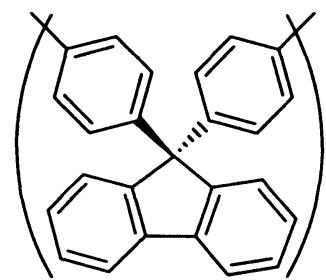

(Xd)

It showed the expected thermal characteristics, $\mathrm{Tg}>300^{\circ} \mathrm{C}$ and $\mathrm{Td}: 562^{\circ} \mathrm{C}$, however, the $k$ value could not be measured since it was not possible to obtain smooth films from amidic solvents, possibly due to the limited solubility. 
Table 2 Solubility of polyarylenes

\begin{tabular}{|c|c|c|c|c|c|c|c|c|c|}
\hline \multicolumn{2}{|c|}{ Polyarylene } & $\begin{array}{c}\text { Entry } \\
\text { No. }\end{array}$ & NMP & CHN & THF & $\mathrm{CHCl}_{3}$ & $\begin{array}{c}\text { Ethyl } \\
\text { lactate }\end{array}$ & Acetone & n-Hex \\
\hline \multicolumn{2}{|c|}{$\mathbf{X a}$} & 1 & - & - & - & - & - & - & - \\
\hline \multicolumn{2}{|c|}{$\mathbf{X b}$} & 2 & - & - & - & - & - & - & - \\
\hline \multicolumn{2}{|c|}{$\mathbf{X c}$} & 3 & - & - & - & - & - & - & - \\
\hline \multicolumn{2}{|c|}{$\mathbf{X d}$} & 4 & + & - & +- & - & - & - & - \\
\hline \multicolumn{10}{|c|}{ Copolymer of Xd with other aromatic monomers } \\
\hline \multicolumn{10}{|c|}{$\begin{array}{cc}\text { comonomer } & \begin{array}{c}\text { composition } \\
\text { molar ratio } \\
\mathbf{X d} / \mathbf{X n}\end{array} \\
\end{array}$} \\
\hline \multirow[t]{2}{*}{$\mathbf{X e}$} & $80 / 20$ & 5 & +- & - & +- & - & - & - & - \\
\hline & $50 / 50$ & 6 & + & - & +- & - & - & - & - \\
\hline \multirow{4}{*}{$\mathbf{X f}$} & $90 / 10$ & 7 & ++ & ++ & ++ & - & - & - & - \\
\hline & $60 / 40$ & 8 & ++ & +- & ++ & - & - & - & - \\
\hline & $30 / 70$ & 9 & ++ & +- & ++ & - & - & - & - \\
\hline & $0 / 100$ & 10 & - & - & - & - & - & - & - \\
\hline \multirow{4}{*}{$\mathbf{X g}$} & $80 / 20$ & 11 & ++ & ++ & ++ & ++ & - & - & - \\
\hline & $50 / 50$ & 12 & ++ & ++ & ++ & ++ & - & - & - \\
\hline & $20 / 80$ & 13 & +- & ++ & ++ & ++ & - & + & - \\
\hline & $0 / 100$ & 14 & - & - & ++ & ++ & - & - & ++ \\
\hline
\end{tabular}

Fluorinated homo-polyarylenes and related polyarylene

\begin{tabular}{cccccccccc}
\hline $\mathbf{X h}$ & 15 & ++ & ++ & - & - & - & - & - \\
\hline $\mathbf{X i}$ & 16 & ++ & - & ++ & ++ & - & - & - \\
\hline $\mathbf{X j}$ & 17 & ++ & ++ & ++ & ++ & ++ & - & - \\
\hline $\mathbf{X k}$ & 18 & +- & - & - & - & - & - & - \\
\hline
\end{tabular}

++ : Soluble at higher $(>10 \mathrm{wt} \%)$ concentration of polymer,

+ : Soluble at $10 \mathrm{wt} \%$ less concentration, +-: Partially soluble, - : Insoluble

In order to enhance solubility we copolymerized Xd with other aromatic monomers. A first attempt with the simple 1,3-phenylene unit (Xe) as co-monomer did not lead to improved properties (entry 5 and 6 in Tables).

Second attempt was to introduce bulky phenyl-substituted aromatic co-monomer (Xf). By introducing 10-40 mol\% of Xf, solubility was<smiles>[X]c1ccc(C2(c3ccc(C)cc3)c3ccccc3-c3ccccc32)cc1</smiles>

$(\mathbf{X d}) /(\mathbf{X e})$ 
significantly improved, leading to an amorphous polymer that is soluble also in cyclohexanone (CHN) (entry 7-9 in Tables).

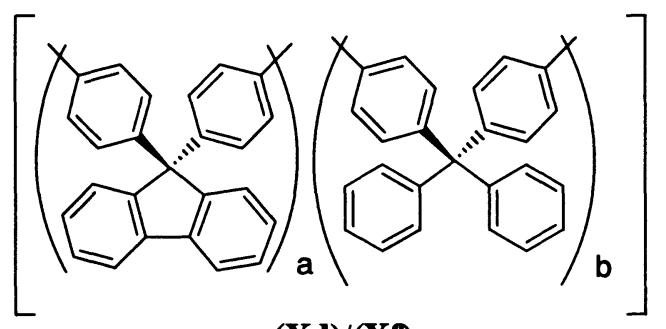

$(\mathbf{X d}) /(\mathbf{X f})$

Homo-poly(Xf) was prepared (entry 10 in Table 1 ), which was expected to have similar solubility, $k$ and thermal stability. However, only an insoluble crystalline polymer formed. The molecular weight was probably low because of the precipitation of polymer during polymerization. Copolymer with $(X d) /(X f)=60 / 40$ on the other hand had sufficient processability for the measurement of the $k$. It exhibited $k=2.7$ (@1MHz), obtained with film coated from CHN solutions. Like poly(Xd) the thermal stability was high, $\mathrm{Tg}:>300^{\circ} \mathrm{C}$, and $\mathrm{Td}: 549^{\circ} \mathrm{C}$. In agreement with our expectation, $k$ is slightly lower than the lowest $k$ of our previously reported non-fluorinated polyimide $(k=2.77$ @1MHz) [4].

In order to get polyarylenes with even lower $k$, we introduced fluorinated units into polymer chain (entry No. 15-18 in Tables).

Co-polymerization of the bismesylate of (Xd) with trifluoromethyl- substituted 1,3-phenylene unit TFDCB (Xg), one of the simplest fluorine-containing commercially available monomer, was investigated (Entry 11-14 in Tables).

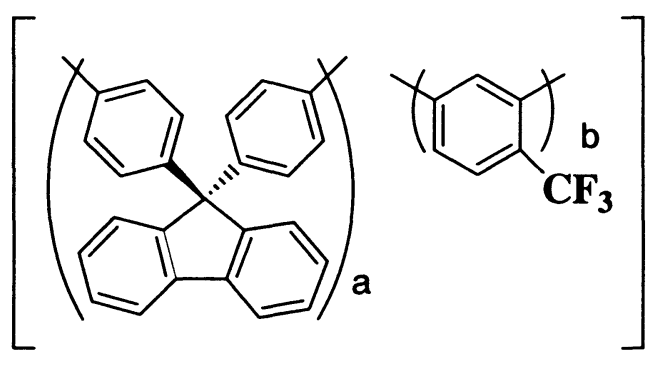

$(\mathbf{X d}) /(\mathbf{X g})$

Substitution with trifluoro-methyl group lead to a major improvement of solubility in comparison to the corresponding non-substituted polymer (entry 5 and 6 in Tables).

Solubility and $k$ changed with the composition of the copolymers. Copolymers with the composition, $(\mathbf{X d} / \mathbf{X g}$ ): 80-50/20-50 (molar ratio) showed $k$ values in the range of 2.5-2.6, significantly lower than those of the non-fluorinated polyarylenes. However, the higher content of $\mathbf{X g}$ in copolymer, the lower was Mw of the copolymer. TFDCB does not have sufficient high reactivity for polymerization (Entry 14 in Table 1).

A promising candidate for polyarylenes with very low $k$ is considered to derive from a monomer with high concentration of fluorine groups.

We studied the polymerization of bismesylate of commercially available fluorine-containing phenols, bis(3-fluoro-4-hydroxyphenyl) diphenylmethane (Xh), $\quad(\mathrm{F}=10.72 \mathrm{wt} \%$ in polymer unit) bis(4-hydroxyphenyl)phenyltrifluoromethylmethane $(\mathrm{Xi})(\mathrm{F}=18.37 \mathrm{wt} \%$ in polymer unit) and Bisphenol AF (Xj) ( $F=37.72 \mathrm{wt} \%$ in polymer unit).<smiles>Cc1ccc(C(c2ccccc2)(c2ccccc2)c2ccc(C)c(F)c2)cc1F</smiles>

(Xh)<smiles>Cc1ccc(C(c2ccccc2)(c2ccc(C)cc2)C(C)(F)F)cc1</smiles>

(Xi)<smiles>[Y]c1ccc(C(c2ccc(C)cc2)(c2ccc(C)cc2)C(F)(F)F)cc1</smiles>

(Xj)

With increasing fluorine content in these polyarylenes, the $\mathrm{Mw}$ of the polymers increased. This is probably due to increased reactivity as a result of the electron-withdrawing effect of fluorine 
groups.

The homopolymers (Xh) and (Xi) had not sufficient high molecular weight to coat them onto As-doped $\mathrm{Si}$ wafer for $k$ measurements. However, the homopolymer derived from Bisphenol AF (Xj) exhibited good solubility and high molecular weight (entry 17 in Table 1). It showed with $k=2.2$ the lowest $k$ and with $\mathrm{Mw}=27000$ the highest $\mathrm{Mw}$ among our polyarylenes. This result was in agreement with previous results of fluorinated fluorenyliden-substituted polyimides containing a bisphenylhexafluoropropane moiety. In that case a dielectric constant of $\mathrm{k}=2.35$ (@1MHz) was obtained, slightly higher due to the polar imide linkage.

The significant improvement in the solubility in polyarylenes with increasing fluorine content was especially remarkable in the comparison between the homopolymer $\mathbf{X j}$ and its non-fluorinated couterpart Xk, Entry No.17 with No.18 (Xk: polyarylene from Bisphenol A), similar to the polymers with a 1,3-phenylene unit (Entry 5-6 and 11-13).

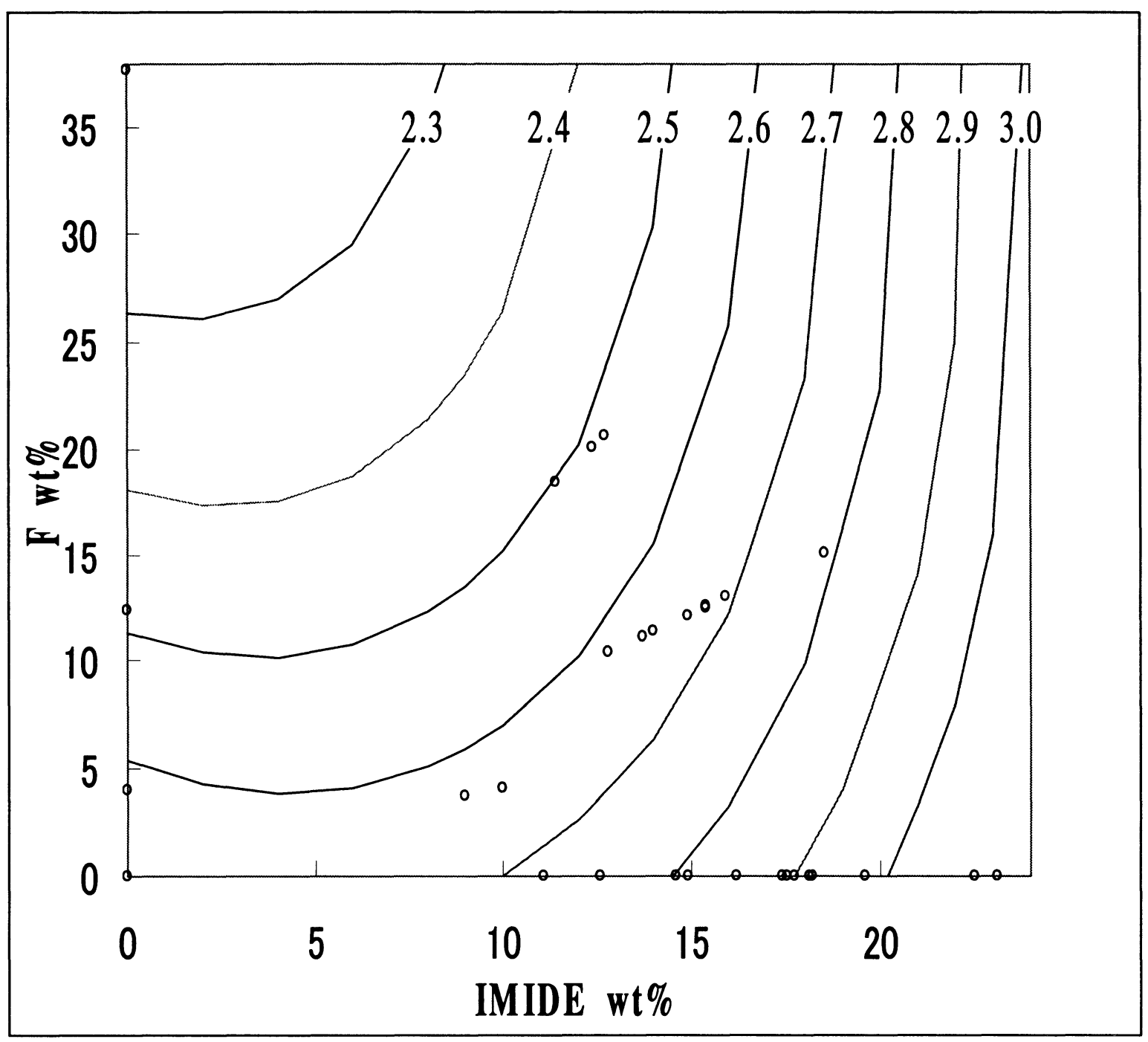

Fig.1 Statistical plots of $\mathrm{k} v$ s. imide concentration and $\mathrm{F}$ content in polyimides and polyarylenes $\circ$ : Experimental data 


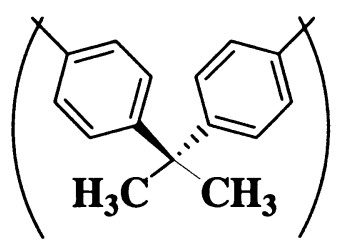

(Xk)

The high moleculaw weight fluorinated homopolymer (Xj) derived from Bisphenol AF was soluble even in ethyl lactate, a solvent recognized as a safe solvent acceptable for microelectronics use. The fluorine-containing polyarylenes formed transparent film as cast from solvents such as $\mathrm{CHN}$, suggesting amorphous nature.

However, with the increase of the fluorine content a drop of $\mathrm{Tg}$ and $\mathrm{Td}$ of polyarylenes was observed. This effect is especially strong in the case of hexafluoroisopropylidene group since the substituent is in the main chain, but the homopolymer from Bisphenol AF still maintains $\mathrm{Tg}=249^{\circ} \mathrm{C}$ and $\mathrm{Td}=508^{\circ} \mathrm{C}$, sufficient high for engineering plastics.

In our investigation of thermally stable polymers with low $k$, polyarylenes have been proven to have reasonable chemical structures for achieving low $k$. Polyarylenes showed lower $k$ values than thermally stable aromatic polyimides containing similar main moieties, $k=2.7$ without fluorine atom and $k=2.2$ with fluorine atom. In other words, our polyimides, $k=2.77$ without fluorine containing groups and $k=2.35$ with fluorine in the polymer, have almost reached the lowest limit for $k$ among polyimides.

Our study of a series of polyimides demonstrated that $k$ values strongly depend on the imide concentration and fluorine content. Combination of the new results of polyarylenes with those of the polyimides gives 31 data for a plot of $k v s$. imide concentration and fluorine content. Using this data set, contour lines of $k$ as a function of imide concentration and fluorine content could be obtained on the basis of statistics with high correlation factor $(f=0.96)$ as shown in Fig. 1. Our results can be used for polymer design of low $k$ materials. They demonstrate that

1) the dielectric constant $k$ value can easily be estimated from repeating units in polyimides and polyarylenes.
2) the requirements for polymer with low dielectric constant. For example, in order to achieve $k$ lower than 2.5, the content of the imide fraction has to be lower than $12 \mathrm{wt} \%$ and the fluorine content higher than $12 \mathrm{wt} \%$.

\section{Conclusions}

We synthesized soluble non-fluorinated polyarylenes with a dielectric constant as low as $k=2.7$, and fluorinated polyarylenes with $k$ as low as $k=2.2$. This is probably the lowest $k$ value achievable for organic bulk polymers with high thermal stability.

Based on the new results from the low $k$ polyarylenes, we estimate that our previously reported polyimides with bulky fluorenylidenemoieties showed the lower limit of $k$ among polyimides, with $k=2.77$ for non-fluorinated and $k=2.35$ for fluorinated polyimides.

Using experimental data of $k$ vs. imide concentration and fluorine content in polymers, we can estimate $k$ value from the chemical structure of repeating unit of polyimides and polyarylenes.

\section{Acknowledgement}

The authors thank to Dr. Igor Rozhanskii, Dr. Takashi Okada and Ms. Mayumi Kakuta-Shinoda for their helpful comments through discussion.

\section{References}

1. K. Goto, M. Kakuta, Y. Inoue and M. Matsubara, J. Photopolym. Sci. Tech., 13, (2000) p.313.

2. K. Goto, M. Kakuta. Y. Inoue and $\mathbf{M}$. Matsubara, Abstract of Polycondensation 2000, , (2000) p.63.

3. K. Goto, M. Kakuta, Y. Inoue and $\mathbf{M}$. Matsubara, Abstract of the $8^{\text {th }}$ Polyimide Conference, (2000) p.9.

4. K. Goto, Y. Inoue, M. Matsubara, J. Photopolym. Sci. Tech.,14, (2001) p.33.

5. V. Percec, S. Okita and R. Weiss, Macromolecules, 25, (1992) p.1816.

6. V. Percec, J-Y. Bae, M. Zhao and D. H. Hill, Macromolecules, 28, (1995) p.6726.

7. V. V. Korshak, S. V. Vinogardova and Y .S. Vygodskii, J. Macromol. Sci., Rev. Macromol. Chem., C11, (1977) p.45. 